\title{
Perspectives of COVID-19 Survivors on the Treatment Experience in Nigeria
}

\author{
Oluremilekun Comfort Kusimo*, Samuel Ogiri, Haruna Ismaila Adamu, Ufuoma Aduh
}

Walden University, Minneapolis, MN, USA

Email: *stremi2001@yahoo.com

How to cite this paper: Kusimo, O.C., Ogiri, S., Adamu, H.I. and Aduh, U. (2022) Perspectives of COVID-19 Survivors on the Treatment Experience in Nigeria. Open Access Library Journal, 9: e8373. https://doi.org/10.4236/oalib.1108373

Received: January 13, 2022

Accepted: February 15, 2022

Published: February 18, 2022

Copyright $\odot 2022$ by author(s) and Open Access Library Inc.

This work is licensed under the Creative Commons Attribution International License (CC BY 4.0).

http://creativecommons.org/licenses/by/4.0/ (c) (i) Open Access

\begin{abstract}
Aim: To explore the perspectives of COVID-19 patients and survivors on the treatment experience and its impact on their lives, socially, psychologically and physically. Background: Nigeria was hit by the COVID-19 pandemic in 2020 with huge impact on an already fragile health system. The impact of the pandemic left many institutions disrupted with pervasive consequences on health, economy and society in general. At the individual level, many affected by the disease were left with lasting negative impacts which were largely unrecognized and trivialized. This paper provides an opportunity to capture the lived experiences of COVID-19 patients and survivors in Nigeria. Method: The study adopted a qualitative, phenomenological design. Semi-structured interviews were conducted digitally from March to August 2021. Fifteen COVID-19 survivors were selected purposively for the interview. Interviews were conducted using electronic platforms such as zoom, e-mails and WhatsApp. The contents of the interviews were transcribed and printed. Data generated was then analyzed manually using the content analysis approach. The respondents included eight males and seven females: including two married couples. Findings: Based on the perceptions of the participants, they experienced stigma and discrimination during their treatment period. Isolation was likened to feeling imprisoned and persons with existing co-morbidities experienced difficulties managing their chronic conditions along with the COVID-19 treatment. The COVID experience generally increased the personal perception of risk as explained by all the participants and it improved adherence to non-pharmaceutical interventions to prevent the COVID-19 infection. Conclusion: Understanding the perceptions of COVID-19 survivors provides an invaluable insight into the treatment experience and how the conditions of treatment impacted patients' treatment and post-treatment outcomes. This deeper understanding may inform policy changes that are targeted at improving patients' care experience and outcomes. Implications for Public Health Practice: Health workers play a key role in the outcome of
\end{abstract}


treatment and should be supported to deliver services in a conducive environment where they themselves feel safe and protected against infections. Testimonials and stories of the survivors may be useful tools to drive positive behavioral change.

\section{Subject Areas}

Infectious Diseases

\section{Keywords}

COVID-19, Survivors, Treatment, Quality of Care

\section{Introduction}

World Health Organization (WHO) declared the human coronavirus disease (SARS-CoV-2) outbreak a Public Health Emergency of International Concern (PHEIC) on January 30, 2020, (Galehdar et al., 2021 [1]; Shi et al., 2020 [2]; WHO, 2020 [3]). The name COVID-19 was coined to reflect the year (2019) the virus was first found in humans (MacKenzie \& Smith, 2020) [4]. Nigeria was identified as one of the 13 high-risk African countries for rapid COVID-19 transmission (WHOAFRO, 2020) [5] and was among the hardest-hit countries based on the burden of COVID-19 (Andam et al., 2020a [6]; Inegbedion, 2021 [7]).

The COVID-19 pandemic has had an overwhelmingly negative impact on the health systems of many countries including Nigeria (Shereen et al., 2020) [8]. It threatens to reverse the gains made so far in achieving universal health coverage and quality of health care in Nigeria. It has caused major disruptions to health services especially at the primary healthcare level (Ahmed et al., 2020) [9]. In resource-limited settings such as Nigeria where health systems are fragile, the impact of the COVID-19 pandemic is enormously grave (Amzat et al., 2020) [10]. The first COVID-19 case was discovered in Ogun State on the $28^{\text {th }}$ of February 2020. Due to the proximity of the state to Lagos, this index case soon moved to Lagos which then quickly became the epicenter of COVID-19 in Nigeria accounting for 50\% of all cumulative cases and deaths (Amzat et al., 2020) [10].

The emergency response was activated by National Centre for Disease Control (NCDC) and a presidential task force was set up to respond to the pandemic with each state having an emergency operating center. The first case was managed at the Lagos state infectious disease isolation center. A national lock-down was set in motion from March 2020 to June 2020. Surveillance was ramped up at all levels including the portals of entry. These measures were effective in flattening the epi-curve, however the impact of an unprecedented pandemic of this scale on human lives remains vaguely quantified.

The COVID-19 pandemic is unprecedented and has created in its wake a trail of peculiar challenges which can only be described as overwhelming to health 
systems (Yang et al., 2020) [11]. The challenges are far reaching and have resulted in economic, social and political disruptions across the globe (Li et al., 2020) [12]. Nigeria is not left out in this global menace, over 223,887 COVID-19 cases have been notified as of December 2021 (NCDC, 2021) [13]. The impact of the pandemic has further weakened the fragile health system of the country despite the huge investments by the government and the private sector to curb the shock of the pandemic (Ahmed et al., 2020) [9]. Non-pharmaceutical and pharmaceutical interventions have been deployed by NCDC; the government agency responsible for health emergencies in the country. Vaccine deployment has proven once again to be a powerful weapon in the fight of epidemics. Non-pharmaceutical interventions deployed in Nigeria are in line with the WHO recommendations and include social distancing measures, wearing of face masks, hand hygiene. Nigeria is not exempted from the infodemic that is trailing the COVID-19 pandemic and several misinformation and disinformation have encumbered the uptake of both pharmaceutical and non-pharmaceutical interventions deployed to control the pandemic.

A gradual flattening and even dip of epi-curves have been observed following the arrival of anti-COVID vaccines, however, the negative impacts of the pandemic persist in many countries including Nigeria. The Nigerian economy has been hard hit by the pandemic, extended lockdowns across the countries led to huge economic losses, disruptions in supply chains and an overall increase in the number of Nigerians who now live below the poverty line of less than 1.90 dollar a day (Amzat et al., 2020 [10]; Andam et al., 2020b [6]; Inegbedion, 2021 [7]). Health inequalities have also worsened as a result of unequal access to health services, lack of health insurance, among others (Ahmed et al., 2020) [9].

There is concern that survivors of COVID-19 infection might be at an increased risk of neurological disorders and psychological sequelae. Given the scale of the pandemic, there were substantial effects on the economy, health and social care systems (Akande-Sholabi \& Adebisi, 2020 [14]; Andam et al., 2020b [6]). Students, private businesses and farmers were disproportionately affected by the COVID-19 pandemic due to restricted movements, uncertainties and weak institutions (Ebuete et al., 2021) [15].

The psychological impact of the COVID pandemic is yet to be fully researched. Current literature regarding the specific cognitive impact of virus-related neurological disease is still quite limited. A study conducted to determine the cognitive and psychological impact of COVID-19 suggested that delirium, insomnia anxieties, depression and post-traumatic stress disorders were common among patients admitted for COVID-19 (Devita et al., 2021) [16]. In some patients, these mental health issues persisted for up to 39 months post contagion. Several studies have predicted negative mental health effects based on the neuropsychological sequelae of previous pandemics like the SARS and Ebola outbreaks (Chakraborty, 2020 [17]; Hawke et al., 2021 [18]; Javed et al., 2020 [19]; Sharma \& Vaish, 2020 [20]; Ueda et al., 2020 [21]). In general, the pan- 
demic was rife with stress and anxieties due to the uncertainties around the COVID-19 disease (Hawke et al., 2021) [18]. Sleep deprivation, fear of infection especially among the vulnerable populations including pregnant women, the elderly and persons with pre-existing mental health conditions are some of the psychological impacts reported (Ueda et al., 2020) [21] Emotional challenges associated with fear of the unknown, proximity to COVID-related deaths and the suffering of those affected have been documented in literature (Ogueji et al., 2021) [22]. Also, social isolation has been identified as a risk factor for poor mental health outcomes (Sharma \& Vaish, 2020) [20].

In view of the far-reaching impact of the COVID-19 pandemic on every sphere of life, it has become pertinent to have a people-centered approach in response to it. Against this backdrop, this study seeks to explore the perspectives of people who have been affected by COVID-19 and how these insights can shape the dynamics between the formal health sector and the populations they serve whilst informing policy changes that can impact positively the quality of care.

\section{Materials and Methods}

\subsection{Study Design}

This study adopted a qualitative methodology to explore the perspectives of persons affected by COVID-19 in Nigeria using the phenomenological-hermeneutic approach. This approach describes the constructs of lived experiences and how they can enable an interpretative understanding of experiential realities that are fundamental to the expression of the essence of the phenomenon (Fuster Guillen, 2019) [23]. This method provides a practical and relational structure to study lived experiences. For this study, semi-structured interviews were conducted virtually for 15 COVID-19 survivors using electronic platforms including zoom, e-mails and WhatsApp.

\subsection{Selection of Participants}

Participants provided oral or written consent before commencing with the interview. All information provided were anonymized to ensure confidentiality was maintained for all respondents. Patients were selected purposively from Lagos, Bauchi and Kano State; and included a range of occupations including health workers, a cleaner, a communication expert, engineers and information technologists. The respondents included 8 males and seven females: including two married couples. Patients were enrolled in the study at the end of their treatment or self-isolation period.

\subsection{Data Collection}

Semi-structured interviews were conducted to generate information in an inductive approach. Question guides were used to generate discussion content in line with the objectives of the study. Data collection commenced in March and 
was concluded in August 2020. To avoid extensive physical contacts with the participants in keeping with the social distancing rules to prevent the transmission of the COVID-19 infection, interviews were conducted using electronic platforms such as zoom, WhatsApp, e-mails and telephone conversations. One of the interviews was conducted in person; for this one, physical distancing and wearing of face masks were observed by both the participant and the interviewer. The question guide was developed by the research team and shared for review among other researchers. The discourse of the interviews was transcribed, and each transcript was stored after removing all unique identifiers.

\subsection{Instrumentation}

A discussion guide was developed for the purpose of the study (Table 1). The interview questions included in the guide were open ended questions and they were used to gather information from the participants. The guide was developed by the research team and reviewed by fellow researchers. Data were gathered with open-ended questions. The main questions in the guide include: 1) What was your experience in general from the time you were infected? 2) Can you share your experience on the medical care you received? 3) Were you comfortable in the treatment center? 4) Were you involved in making decisions about your care? 5) Based on your recent experience while on treatment, what are the things that should have been done differently? 6) Post treatment, what are your immediate concerns?

Other probing questions include: What were your initial symptoms, if any? Any history of travel or contact with a confirmed COVID-19 case prior to onset of symptoms? How were you diagnosed? How was the result communicated and what was your reaction (response)? Was any member of your family or close contacts infected?

All interviews were conducted in English Language hence translation was not required.

\subsection{Data Analysis}

The content of the interviews was transcribed and printed. Data generated was then analyzed manually using the content analysis approach. The analysis was rigorous using the Granheim and Lundman's 5-step methods recommended (Graneheim \& Lundman, 2004) [24]. The initial step involved the stepwise transcription of the interviews, this was followed by an initial immersion in the texts and interview recordings. The third step was a systematic analysis of the content to identify the threads of meanings that were related and then coding to enable a logical stringing of similar meanings. Sub-themes were then identified and categorized, in the fourth step, to give structure to the analysis. In the final stage of the analysis, sub-themes were crystallized into key concepts or themes in line with the content of the texts. 
Table 1. Discussion guide.

\section{DISCUSSION GUIDE FOR COVID-19 KEY INFORMANT VIRTUAL INTERVIEW}

\section{Consent: Do you provide consent to document, use, store and share the information provided for reporting and communication purposes?}

\section{$\mathrm{X} \square$ YES $\square$ NO}

\section{S/N Question}

1 I am aware that you have recently tested positive for COVID-19. What were your initial symptoms, if any? Any history of travel or contact with a confirmed COVID-19 case prior to onset of symptoms? How were you diagnosed? How was the result communicated and what was your reaction (response)? Was any member of your family or close contacts infected?

Were you admitted in the treatment centre? If yes, how long did it take between receiving the result and being taken for admission? How were you evacuated and from where??

Can you share your experience on the medical care you received? ?

2 What were the attitudes of the hospital staff that provided you with medical care and support?

What were the admission formalities? Were you asked to provide consent? Were you attended to promptly? Can you recall the medications you were placed on? What other services were rendered to you?

3 Were you comfortable in the treatment centre? If yes, describe the positive attributes of the centre and the unfavourable ones. How did you overcome them?

4 Were you treated with respect? Describe. Was there a reason to complain about anything and to whom?

5 Were you involved in making decisions about your care? If yes, to what extent did that meet your expectations?

6 How did the doctors, nurses and other support staff communicate with you while in isolation?

7 Based on your recent experience while on treatment, what are the things that should have been done differently?

8 How would you describe the relationship you have with the care providers at the facility?

9 What would you say are the most important issues you would like to be improve about your treatment experience? Post treatment, what are your immediate concerns?

10 Based on your recent experience, what advice would you give to those who are still sceptical about COVID-19?

11 Post-treatment, did you have difficulties resuming normal daily activities and life in general? Did you experience discrimination in the community due to your COVID-19 status? What would you be doing differently?

12 Are there any other comments you would like to add?

\subsection{Ethical Considerations}

Enrolment into the study was completely voluntary. Participants were informed of their rights to discontinue with the interview if they were no longer comfortable with the research procedures. All participants provided either verbal or written informed consent before the interview. All information provided were de-identified before data analysis to ensure both confidentiality and anonymity.

\section{Results}

Transcripts of interviews were analyzed manually using the using the Granheim and Lundman's 5-steps method. After reading and re-reading the transcripts, 
themes and sub-themes were identified. The data was then interpreted in-depth by the lead researcher. The interpretations were shared and discussed with the other three researchers involved in the study.

Eight males and seven females with an average age above 35 years old. The lived experiences of the respondents were abstracted from the texts and categorized into four broad themes including health-system related factors, feeling of confinement, post-COVID trauma and COVID-related anxiety. The sub-themes included perceived discrimination by the survivors, feeling of neglect during treatment, delay in getting results, difficulties in navigating the health system, prison-like feeling while in isolated care, weak follow up of infected individuals and various forms of anxieties that stemmed from the COVID-19 experience. See Table 2 for details regarding the main and sub-themes as well as insightful quotes from the respondents.

\subsection{Health System-Related Factors}

A few of the patients reported that they experienced health-worker related stigmatization that affected them psychologically. A respondent stated that he felt like he was being avoided by the nurses and clinicians. "Whenever they came around, they were covered from head to toe and that made me feel very contagious". Discrimination of infectious diseases due to fear of getting infected while caring for patients was a key concept identified.

Difficulties in navigating the health system and getting prompt treatment were identified by one of the respondents as one of the factors responsible for high mortality rates among the severe cases. Quoting the respondent directly, "a friend of mine said get yourself to the COVID-19 treatment center quickly, this disease is time-bound". A survivor who was enrolled for home-based care suggested very weak follow-up by the health system after a positive result; "no one contacted us while we were isolating at home".

\subsection{Confinement/Isolation}

Separation from family members was identified by the respondents who were in isolation as the most difficult part of the process. Very few of the patients mentioned that they enjoyed the solitude and took the opportunity to rest, reflect and meditate. Isolation was mostly long, lonely and intense. A respondent compared the experience to being in the prison. Patients spent hours and days alone, meeting a few nurses or doctors for short time, which increased their suffering and feelings of loneliness. Also, physical activities were limited while in the treatment wards. Quoting one of the respondents "I could not go for my walks".

\subsection{Post-COVID Trauma}

A respondent experienced discrimination post-treatment by neighbors; this was explained by the refusal to interact with the respondent's family members by the immediate neighbors who believed that the family members still carried the 
Table 2. Main and sub-themes; concepts relevant for public health practice.

\begin{tabular}{lll}
\hline S/N Main Theme & Sub-theme \\
\hline $1 \quad$ Health-system & Perceived discrimination of infectious \\
related factors & $\begin{array}{l}\text { diseases due to fear of getting infected } \\
\text { while caring for patients }\end{array}$
\end{tabular}

\section{Quotes \& Extracted Concepts}

“...Nobody wants to associate with you or touch what you have touched, when I was really sick, an expert doctor was called to review me but to my surprise the other doctors were telling him to leave"

Feeling of neglect among patients due to Patients felt they were being avoided by the health workers who health workers' fear of getting infected were expected to attend to them due to fear of getting infected.

Delay in getting results

Difficulty in navigating the healthcare system to access COVID services

Weak follow-up of confirmed COVID-19 cases.

2 Confinement/ Prison-like treatment of patients Isolation

Limited social activities

3 Post-COVID Discrimination and stigmatization by trauma people both literate and illiterate.

Fear of re-infection.

Fear of infecting family members. Paranoia and astute awareness of the pandemic.

4 COVID-relate Fear of the unknown $\mathrm{d}$ anxieties

Death anxiety

Source of infection unknown

Fear of re-infection.

Heightened perception of self-risk

Fear of aggravated co-morbidities
"I had to lobby through a colleague for the sample to be taken and the result was not communicated on time.

Frustration from delay in getting follow-up results

"My experience at the testing site was chaotic.... they were rude and yelling at my son". Complicated pathways to getting tested and retrieving test results

Not all patients received medicines. Family members were not trained on infection, prevention and control in many cases and some patients isolating in their homes were not followed up by the response team or were reached over the phone after their treatment was almost over.

"Feeling like a prisoner with no access to sunlight" "I was alone in my room; I was in prison"

Inability to engage in physical activities required for management of existing co-morbidities

"I sensed discriminatory reaction...even by elites who think they are literate"

"I cannot sleep, and I feel like I have been reinfected again"

Fear of getting re-infected was stated by many and some sought professional psychological help.

"I feel devastated and scared"

Many of the patients were not able to identify the source of infection. This created fear and uncertainty on how to protect themselves from getting re-infected

"This is an experience I can never forget.... COVID is real and it's everyone's responsibility to protect self and others"

Patients were more careful and wanted people to know that COVID-19 was real and that it was a fatal disease

Survivors were more aware of others' complacency in observing safety precautions and condemned it.

"Need for exercises to keep us in shape"

Patients with co-morbidities experienced challenges while in isolation.

Some expressed that adequate care was not provided while in isolation; a patient stated that the caregivers did not pay attention to his blood pressure while in isolation. 
disease. Other patients mentioned that they were received with warmth post-treatment without discrimination from friends and work colleagues.

Resumption to work and regular daily activities was difficult for many of the patients that experienced severe form of COVID-19. Some reported sequelae of these symptoms long after they were declared cured of COVID-19. One of the respondents explained that having a therapist helped in the recovery process, another reported daily physical activities like walking and jogging aided his recovery. Also, some patients still experienced respiratory symptoms after discharge. Other long-term sequelae include pain, myalgia, fatigue depression and subjective feeling of isolation.

\subsection{COVID-Related Anxieties}

In this study, many of the confirmed COVID-19 patients were asymptomatic. Those who had symptoms complained of fever, feeling of warmth around the chest area that was radiating to the stomach, cold and hot spells, chest pain, loss of sense of smell, headaches, myalgia and a general feeling of malaise. Very few of the cases were severe enough to experience low levels of oxygen, for those respondents, they expressed deep feeling of fear.

Many of the respondents expressed their frustration at people who are yet to adopt non-pharmaceutical interventions such as wearing of face masks and social distancing. Respondents were also more predisposed to educate others about COVID-19 and the preventive measures. Survivors of the COVID-19 virus were more likely to have higher self-perception of risk. This behavioral outcome can be explored to increase awareness about the risk of COVID-19, impact on health and other facets of life and how to prevent getting infected.

In patients admitted to the intensive care unit, fear of dying was intense. Coupled with the anxiety, patients felt ostracized and stigmatized. Some patients felt the fear of being contagious even after hospital discharge.

Having other co-morbidities complicated the COVID-19 treatment for some of the patients. A few of them mentioned increase in their blood pressure from the anxiety related to the disease. Another respondent stated that medications for existing co-morbidities were not readily available in the treatment centre. Not knowing the medication given for COVID treatment was expressed as a cause of concern by a respondent. Other discomforts described by the respondents include social isolation, sleep problems, disturbance from other patients and generalized meal plan without considerations for special dietary needs and individual preferences.

\section{Discussion}

The findings included four main themes; health system-related factors, confinement/isolation, post-COVID trauma and COVID-related anxieties were described by the survivors of the COVID-19 disease. Health workers were identified as key contributors to the outcome of treatment by the respondents. Dis- 
crimination by the health workers affected the patients psychologically. This corroborates the findings by Ventura et al which suggest that patients felt a sense of well-being when health workers reassured them (Venturas et al., 2021) [25]. Feeling of loneliness, helplessness and isolation was experienced while in the isolation or treatment ward (Jamili et al., 2021) [26]. Like previous studies, this was linked with the feeling of abandonment, negative psychological effects and ultimately poor health outcomes (Ashley, James, Williams, et al., 2021 [27]; Peng et al., 2021 [28]; Sharma \& Vaish, 2020 [20]).

Several studies suggest that the heavy burden placed on healthcare workers by the COVID-19 pandemic may be responsible for feeling of fear among them (Ashley, James, Stephen, et al., 2021 [29]; Chegini et al., 2021 [30]; Searby \& Burr, 2021 [31]; Vázquez-Calatayud et al., 2021 [32]). Fear may play out as discrimination particularly if the healthcare professionals feel exposed to the COVID infection due to inadequate personal protective equipment (PPE) supplies and other infection, prevention control limitations as explained in previous studies (Ashley, James, Williams, et al., 2021 [27]; Chegini et al., 2021 [30]). Hence, psycho-social support is necessary for both patients and the healthcare workers as well as adequate supply of PPEs (Ogueji et al., 2021 [22]; Peng et al., $2021[28])$.

One of the respondents explained the need to use a private health facility in order to access prompt care. This is suggestive of a basic lack of trust in the public health facility due to regular shortages of oxygen and other resource limitations. Delay in getting test results was also described as an issue. Poor follow up of patients who were isolating in their homes was a key concern as it uncovers gaps in the health system which may impact on quality of care as well as overall COVID-19 surveillance.

All respondents expressed some form of anxiety. This is similar to the findings in several other studies that explored the lived experiences of COVID patients (Galehdar et al., 2021 [1]; Venturas et al., 2021 [25]). These anxieties had negative impact on the patients' outcomes. Coping mechanisms were developed to navigate the treatment; they include prayers, talking to loved ones and meditating. In contrast to the findings by Maunder et al., no survivor mentioned anger as one of the emotions experienced during their treatment. Isolation was mentioned to have increased the risk of mental disorders such as anxiety, mood, and suicidal thought as well as acute and post-traumatic stress disorders.

Post-COVID, patients experienced fear of getting re-infected, stigma and fear of unknown after-effects of the disease, among others. This heightened sense of perceived risk made the respondents more likely to adopt the non-pharmaceutical interventions such as wearing of face masks and social distancing to prevent the infection. They were also more likely to condemn those who were non-compliant. Lasting psychological impact of the disease may require professional psychological support in some cases. In general, post-COVID care appeared to be necessary for all patients after their discharge from treatment. Care may need to address physical therapy, mental health and rehabilitative care as suggested by 
(Ogueji et al., 2021 [22]; Stephens \& Breheny, 2021 [33]).

The framework in Figure 1 gives a summary of the findings of this study and their interaction with the constructs of behavioral change.

\section{Limitations}

This study seeks to describe the experiences of the survivors of COVID-19 in Nigeria based on their own perspectives. The strength of the study is that unlike many of other studies that were focused on the experience of the healthcare professionals, the focus was on the patients who experienced the disease. The sample size was limited due to the challenges of conducting interviews within the context of the COVID-19 social restrictions. Similarly, the use of electronic platforms for interviews may have limited the flow of information from the respondents. Inability to engage actively with the respondents and follow their non-verbal clues through the electronic platforms made it impossible to extract meanings beyond the spoken words.

There is a long-time lapse between the conduct of the interviews and the reporting of the findings. This may affect the currency of some of the findings in view of the dynamic nature of the COVID-19 virus and how this may impact on the patients' experience.

Lastly, an in-depth interview would have better captured the lived experiences of the COVID-19 survivors, however, in view of the COVID-19 social restrictions, this was not practical as interviews could not be conducted face to face.

\section{Conclusion}

Due to the burden of COVID-19 in Nigeria, it is critical to capture the essence of the lived experiences of COVID-19 survivors. Understanding the perceptions of

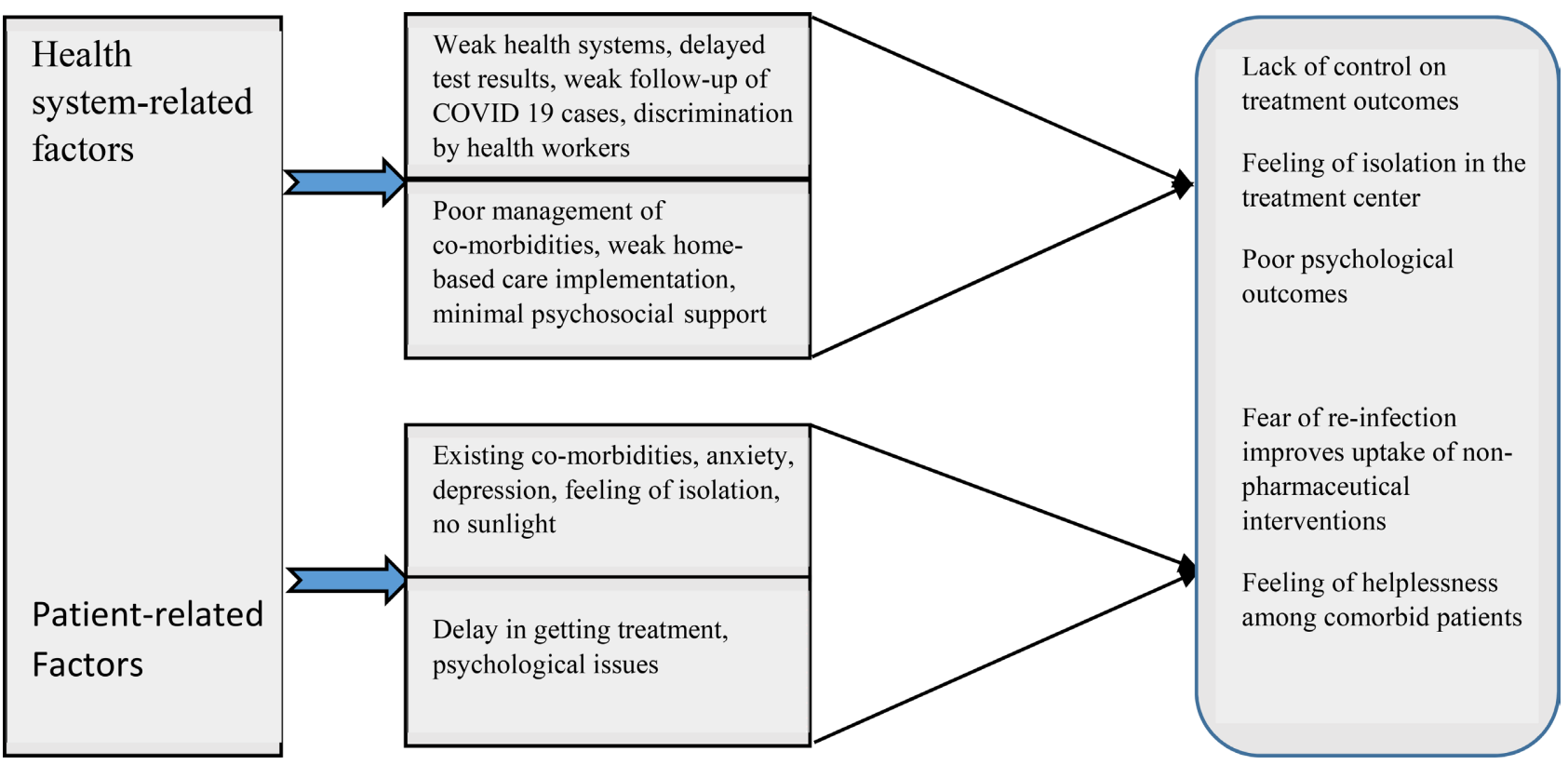

Figure 1. Behavioral framework. 
COVID-19 survivors provides an invaluable insight into the treatment experience and how the conditions of treatment impacted patients' treatment and post-treatment outcomes. This deeper understanding may inform policy changes that can impact positively the quality of care and improve patients' care experience and outcomes.

\section{Implications for Future Studies}

For future studies, it is pertinent to investigate the social factors that drive the COVID-19 pandemic, and the behavioral patterns that are associated with low uptake of non-pharmaceutical and pharmaceutical interventions.

\section{Conflicts of Interest}

The authors declare no conflicts of interest.

\section{References}

[1] Galehdar, N., Toulabi, T., Kamran, A. and Heydari, H. (2021) Exploring Nurses' Perception of Taking Care of Patients with Coronavirus Disease (COVID-19): A Qualitative Study. Nursing Open, 8, 171-179. https://doi.org/10.1002/nop2.616

[2] Shi, Y., Wang, G., Cai, X.P., Deng, J.W., Zheng, L., Zhu, H., Zheng, M., Yang, B. and Chen, Z. (2020) An Overview of COVID-19. Journal of Zhejiang University: Science B, 21, 343-360. https://doi.org/10.1631/jzus.B2000083

[3] World Health Organization (2020) WHO COVID 19 Situation Report. https://www.who.int/docs/default-source/coronaviruse/situation-reports/20200124sitrep-4-2019-ncov.pdf?sfvrsn=9272d086 8

[4] MacKenzie, J.S. and Smith, D.W. (2020) COVID-19: A Novel Zoonotic Disease Caused by a Coronavirus from China: What We Know and What We Don't. Microbiology Australia, 41, 45-50. https://doi.org/10.1071/MA20013

[5] WHOAFRO (2020) SITREP_COVID-19_WHOAFRO_20200311-eng. World Health Organization AFRO.

[6] Andam, K., Edeh, H., Oboh, V., Pauw, K. and Thurlow, J. (2020) Impacts of COVID-19 on Food Systems and Poverty in Nigeria. Advances in Food Security and Sustainability, 15, 145-173. https://doi.org/10.1016/bs.af2s.2020.09.002

[7] Inegbedion, H. (2021) Impact of COVID-19 on Economic Growth in Nigeria: Opinions and Attitudes. Heliyon, 7, e06943.

https://doi.org/10.1016/j.heliyon.2021.e06943

[8] Shereen, M.A., Khan, S., Kazmi, A., Bashir, N. and Siddique, R. (2020) COVID-19 Infection: Origin, Transmission, and Characteristics of Human Coronaviruses. Journal of Advanced Research, 24, 91-98. https://doi.org/10.1016/j.jare.2020.03.005

[9] Ahmed, S.A.K.S., Ajisola, M., Azeem, K., Bakibinga, P., Chen, Y.F., Choudhury, N.N., Fayehun, O., Griffiths, F., Harris, B., Kibe, P., Lilford, R.J., Omigbodun, A., Rizvi, N., Sartori, J., Smith, S., Watson, S.I., Wilson, R., Yeboah, G., Aujla, N., Yusuf, R., et al. (2020) Impact of the Societal Response to Covid-19 on Access to Healthcare for Non-Covid-19 Health Issues in Slum Communities of Bangladesh, Kenya, Nigeria and Pakistan: Results of Pre-Covid and Covid-19 Lockdown Stakeholder Engagements. BMJ Global Health, 5, e003042.

https://doi.org/10.1136/bmigh-2020-003042 
[10] Amzat, J., Aminu, K., Kolo, V.I., Akinyele, A.A., Ogundairo, J.A. and Danjibo, M.C. (2020) Coronavirus Outbreak in Nigeria: Burden and Socio-Medical Response during the First 100 Days. International Journal of Infectious Diseases, 98, 218-224. https://doi.org/10.1016/j.ijid.2020.06.067

[11] Yang, L., Liu, S., Liu, J., Zhang, Z., Wan, X., Huang, B., Chen, Y. and Zhang, Y. (2020) COVID-19: Immunopathogenesis and Immunotherapeutics. Signal Transduction and Targeted Therapy, 5, Article No. 128. https://doi.org/10.1038/s41392-020-00243-2

[12] Li, T., Lu, H. and Zhang, W. (2020) Clinical Observation and Management of COVID-19 Patients. Emerging Microbes and Infections, 9, 687-690. https://doi.org/10.1080/22221751.2020.1741327

[13] NCDC (2021) National Centre for Disease Control COVID-19 Situation Report|COVID-19 Situation Report Weekly Epidemiological Report 62 State of the Natio.

https://ncdc.gov.ng/diseases/sitreps/?cat=14\&name=An\%20update $\% 20$ of $\% 20 \mathrm{COVI}$ D-19\%20outbreak\%20in\%20Nigeria

[14] Akande-Sholabi, W. and Adebisi, Y.A. (2020) The Impact of COVID-19 Pandemic on Medicine Security in Africa: Nigeria as a Case Study. Pan African Medical Journal, 35, 73. https://doi.org/10.11604/pamj.supp.2020.35.2.23671

[15] Ebuete, A.W., Berezi, O.K., Ndiwari, L.E. and Isiya, S. (2021) Domino Effect of Coronavirus in Nigeria. An Overview of the Socioeconomic, Religious and Educational Perspectives. OALib, 8, 1-14. https://doi.org/10.4236/oalib.1106967

[16] Devita, M., di Rosa, E., Iannizzi, P., Bianconi, S., Contin, S.A., Tiriolo, S., Bernardinello, N., Cocconcelli, E., Balestro, E., Cattelan, A., Leoni, D., Mapelli, D. and Volpe, B. (2021) Cognitive and Psychological Sequelae of COVID-19: Age Differences in Facing the Pandemic. Frontiers in Psychiatry, 12, Article ID: 711461. https://doi.org/10.3389/fpsyt.2021.711461

[17] Chakraborty, N. (2020) The COVID-19 Pandemic and Its Impact on Mental Health. Progress in Neurology and Psychiatry, 24, 21-24. https://doi.org/10.1002/pnp.666

[18] Hawke, L.D., Monga, S., Korczak, D., Hayes, E., Relihan, J., Darnay, K., Cleverley, K., Lunsky, Y., Szatmari, P. and Henderson, J. (2021) Impacts of the COVID-19 Pandemic on Youth Mental Health among Youth with Physical Health Challenges. Early Intervention in Psychiatry, 15, 1146-1153. https://doi.org/10.1111/eip.13052

[19] Javed, B., Sarwer, A., Soto, E.B. and Mashwani, Z.R. (2020) The Coronavirus (COVID-19) Pandemic's Impact on Mental Health. International Journal of Health Planning and Management, 35, 993-996. https://doi.org/10.1002/hpm.3008

[20] Sharma, N. and Vaish, H. (2020) Impact of COVID-19 on Mental Health and Physical Load on Women Professionals: An Online Cross-Sectional Survey. Health Care for Women International, 41, 1255-1272. https://doi.org/10.1080/07399332.2020.1825441

[21] Ueda, M., Stickley, A., Sueki, H. and Matsubayashi, T. (2020) Mental Health Status of the General Population in Japan during the COVID-19 Pandemic. Psychiatry and Clinical Neurosciences, 74, 505-506. https://doi.org/10.1111/pcn.13105

[22] Ogueji, I.A., Agberotimi, S.F., Adesanya, B.J. and Gidado, T.N. (2021) Mental Health and Coping Strategies during the COVID-19 Pandemic: A Qualitative Study of Unemployed and Employed People in Nigeria. Analyses of Social Issues and Public Policy, 21, 941-959. https://doi.org/10.1111/asap.12259

[23] Fuster Guillen, D.E. (2019) Investigación cualitativa: Método fenomenológico hermenéutico. Propósitos y Representaciones, 7, 201. 
https://doi.org/10.20511/pyr2019.v7n1.267

[24] Graneheim, U.H. and Lundman, B. (2004) Qualitative Content Analysis in Nursing Research: Concepts, Procedures and Measures to Achieve Trustworthiness. Nurse Education Today, 24, 105-112. https://doi.org/10.1016/j.nedt.2003.10.001

[25] Venturas, M., Prats, J., Querol, E., Zabalegui, A., Fabrellas, N., Rivera, P., Casafont, C., Cuzco, C., Frías, C.E., Olivé, M.C. and Pérez-ortega, S. (2021) Lived Experiences of Hospitalized Covid-19 Patients: A Qualitative Study. International Journal of Environmental Research and Public Health, 18, 10958. https://doi.org/10.3390/ijerph182010958

[26] Jamili, S., Ebrahimipour, H., Adel, A., Badiee aval, S., Hoseini, S.J., Vejdani, M. and Ebnehoseini, Z. (2021) Experience of Patients Hospitalized with COVID-19: A Qualitative Study of a Pandemic Disease in Iran. Health Expectations, 5, 1-9. https://doi.org/10.1111/hex.13280

[27] Ashley, C., James, S., Williams, A., Calma, K., Mcinnes, S., Mursa, R., Stephen, C. and Halcomb, E. (2021) The Psychological Well-Being of Primary Healthcare Nurses during COVID-19: A Qualitative Study. Journal of Advanced Nursing, 77, 3820-3828. https://doi.org/10.1111/jan.14937

[28] Peng, X., Yang, Y., Gao, P., Ren, Y., Hu, D. and He, Q. (2021) Negative and Positive Psychological Experience of Frontline Nurses in Combatting COVID-19: A Qualitative Study. Journal of Nursing Management, 13, 1-9. https://doi.org/10.1111/jonm.13481

[29] Ashley, C., James, S., Stephen, C., Mursa, R., McInnes, S., Williams, A., Calma, K. and Halcomb, E. (2021) Primary Health Care Nurses' Perceptions of Risk during COVID-19: A Qualitative Study. Journal of Nursing Scholarship, 53, 689-697. https://doi.org/10.1111/jnu.12698

[30] Chegini, Z., Arab-Zozani, M., Rajabi, M.R. and Kakemam, E. (2021) Experiences of Critical Care Nurses Fighting against COVID-19: A Qualitative Phenomenological Study. Nursing Forum, 56, 571-578. https://doi.org/10.1111/nuf.12583

[31] Searby, A. and Burr, D. (2021) The Impact of COVID-19 on Alcohol and Other Drug Nurses' Provision of Care: A Qualitative Descriptive Study. Journal of Clinical Nursing, 30, 1730-1741. https://doi.org/10.1111/jocn.15732

[32] Vázquez-Calatayud, M., Rumeu-Casares, C., Olano-Lizarraga, M. and Regaira Martínez, E. (2021) Nursing Students' Experience of Providing Frontline COVID-19 Support: A Qualitative Study. Nursing and Health Sciences, 10, 1-9. https://doi.org/10.1111/nhs.12902

[33] Stephens, C. and Breheny, M. (2021) Diverse Experiences among Older Adults in Aotearoa/New Zealand during COVID-19 Lockdown: A Qualitative Study. Australasian Journal on Ageing, 12, 10-14. https://doi.org/10.1111/ajag.12995 